\title{
Analysis of the Sagittal Balance in Patients with Adult Spinal Deformity: Modified Sagittal Vertical Axis
}

\section{Yang Yu}

Nankai University School of Medicine

\section{Bing Wu}

301 Military Hospital: Chinese PLA General Hospital

\section{Zhaohan Wang}

301 Military Hospital: Chinese PLA General Hospital

Junyao Cheng

301 Military Hospital: Chinese PLA General Hospital

\section{Bo Li}

301 Military Hospital: Chinese PLA General Hospital

\section{Jinjin Liu}

301 Military Hospital: Chinese PLA General Hospital

\section{Kai Song}

301 Military Hospital: Chinese PLA General Hospital

Zheng Wang ( $\square$ wangzheng301@163.com)

301 Military Hospital: Chinese PLA General Hospital

\section{Research article}

Keywords: sagittal balance, adult spinal deformity, quality of life, SVA

Posted Date: June 28th, 2021

DOI: https://doi.org/10.21203/rs.3.rs-599654/v1

License: (1) (1) This work is licensed under a Creative Commons Attribution 4.0 International License. Read Full License 


\section{Abstract}

Objective: The overall sagittal balance of patient with adult spinal deformity (ASD) is crucial for satisfactory postoperative outcome. SVA is the parameter often used to assess the sagittal balance. However, pelvis often rotates backward to compensate for sagittal imbalance in patients with ASD. In the case, SVA cannot reflect the real sagittal balance. Modified sagittal vertical axis (MSVA) is the parameter we found to better assess the real sagittal balance in patients with ASD. And we want to explore the relationship between MSVA and the quality of life.

Methods: We used sample of 60 patients with ASD who underwent long-segment orthopedic surgery ( $\geq 4$ vertebrae) between the period of 2015 and 2018. The paired-sample t test and Pearson correlation analysis were used for statistical analysis. $\mathrm{P}$ value $<0.05$ was considered significant.

Results: SVA, TK, TLK, LL, SS, PT, PI-LL and MSVA were significantly changed in 60 patients preoperatively and postoperatively $(p<0.05)$. The ODI, SRS22, VAS scores were significantly improved postoperatively $(p<0.05)$. Pearson correlation analysis was used to conclude that postoperative SVA, postoperative MSVA and postoperative quality of life score were related. Postoperative MSVA has the strongest correlation with the quality-of-life score.

Conclusions: Through spinal orthopedic surgery, the postoperative spinal parameters and quality of life of patients with ASD were significantly improved. MSVA is of great significance for evaluating postoperative sagittal balance and quality of life of patients with ASD.

\section{Instruction}

Adult spinal deformity (ASD) is a complex spine disease that involves changes in human bones, muscles, and biomechanics [1-3]. Generally, it is more common in women. For patients with ASD, sagittal balance has a greater impact on clinical symptoms than coronal balance [4-5]. In 2009, Schwab et al. proposed that a satisfactory spine sagittal alignment determines satisfactory postoperative function. Among them, the sagittal vertical axis (SVA) is particularly important for the evaluation of the patient's sagittal alignment, and is closely related to the postoperative function of the patient [6]. However, in patients with ASD, pelvis often rotate backward to compensate. In the case, SVA is a compensated value, which cannot objectively reflect the real sagittal imbalance of patients. Therefore, it is important to find a new parameter to evaluate both sagittal balance and pelvis compensation--the modified sagittal vertical axis(MSVA). This study aims to compare the changes in spine-pelvic parameters after orthopedic surgery in patients with ASD, and to figure out the relationship between MSVA and quality of life in patients with ASD.

\section{Methods}

\section{Some important concepts}


TPT-LINE: Vialle et al. obtained the formula PT $=0.37 * \mathrm{PI}-7$ to predict the theoretical PT value (TPT), which means the real PT value without pelvis compensation [7]. The difference between PT and TPT is defined as $\triangle \mathrm{PT}$, which can reflect the pelvis compensation. According to the theoretical PT value, the line drawn from the midpoint of the hip axis is called TPT-LINE (Fig.1).

PNP-LINE: PNP-LINE (Pelvis neutral position line) is the parallel line of TPT-LINE drawn from the posterior superior corner of the sacrum (Fig.2).

SVA囚the distance from $\mathrm{C} 7$ to the posterior superior corner of the sacrum is defined as SVA, and can reflect the sagittal balance (Fig.2).

MSVA: The distance from C7 to PNP-LINE is called the modified sagittal vertical axis (MSVA), which can reflect the real sagittal imbalance. (Fig.2).

\section{The samples of patients}

This was a single-institution retrospective study. A total of 60 patients with ASD treated in our hospital from January 2015 to December 2018 were included in this study. The visual analog scale (VAS), Oswestry Disability Index (ODI), and SRS22 questionnaire were used to assess HRQOL. (It is worth mentioning that, according to the actual situation of follow-up, the authors deleted the 5 Questions 4, 6 , $10,14,19$ of the self-image evaluation part in the SRS22 score, because many patients with ASD are older and cannot answer these questions well, the authors deleted Question 17, because many patients with ASD no longer go to work or school. The authors also deleted question 21 and 22 to ensure the consistency of preoperative and postoperative scores. Therefore, the SRS22 score in this article is a variant of the SRS22 score, which focuses more on the assessment of pain and function).

This clinical study is a retrospective study. It only collects clinical data of patients, does not interfere with the treatment plan of patients, and will not bring risks to the physiology of patients. The researchers will try their best to protect the information provided by patients from disclosing personal privacy, hereby apply for exemption from Institutional Review Board approval.

\section{Imaging parameter measurement}

All patients underwent standard standing full-length poster-anterior and lateral X-rays before and after the operation. The measurements were completed by the same experienced researcher twice and averaged to reduce error. The following parameters were measured in the surgimap system. Radiographic parameter: lumbar lordosis (LL), thoracic kyphosis (TK), pelvic incidence (PI), pelvic tilt (PT), sacral slope (SS), SVA and MSVA.

\section{Statistical analysis}

SPSS22.0 software was used for statistical analysis. First, a paired-sample $t$ test was performed on the spine-pelvic parameters and the quality of life score preoperatively and postoperatively. Then, the 
postoperative ODI and SRS22 scores and the spine-pelvic parameters were analyzed by Pearson correlation. The difference was statistically significant when $p<0.05$.

\section{Results}

The 60 patients in this study all underwent posterior spinal decompression and internal fixation orthopedics, with an average fixed segment of $8.15 \pm 2.22$. Among them, 48 patients $(48 / 60)$ had the head end fixed to the thoracic spine, and 42 patients had the caudal end fixed to the pelvis, of which 20 patients $(20 / 60)$ were fixed to the $S 1$ and 22 patients were fixed to the $S 2(22 / 60)$.

SVA, TK, TLK, LL, SS, PT, PI-LL and MSVA were significantly changed in 60 patients preoperatively and postoperatively (Table1). The ODI, SRS22, low back pain VAS and leg pain VAS scores were significantly improved (Table2).

Pearson correlation analysis was used to analyze the relationship between preoperative sagittal parameters and preoperative quality of life scores (Table 3 ). There is a strong correlation between postoperative sagittal parameters and postoperative quality of life scores (Table4).

\section{Discussion}

Adult spinal deformity (ASD) is a complex spine disease. It is generally believed that the balance of the coronal and sagittal planes has a clear correlation with the patient's quality of life. Among them, sagittal balance has a greater impact on the quality of life of patients with ASD [8, 9]. At present, the evaluation of sagittal balance is mainly accomplished by measuring SVA. It is generally believed that SVA less than 5 $\mathrm{cm}$ can be considered as sagittal balance [6]. However, in patients with ASD, pelvis often rotate backward to compensate the imbalance trend. When a healthy adult stand in a natural standing position, pelvis is in the neutral position. For patients with ASD, due to lumbar lordosis decreased and thoracic kyphosis increased, their body shifts forward. However, to regain trunk balance and reduce energy consumption, the pelvis will rotate backward to compensate. In the case, SVA is a compensated value, which cannot objectively reflect the real sagittal imbalance and the real function. Therefore, it is important to find a new parameter to evaluate both sagittal balance and pelvis compensation--the modified sagittal vertical axis(MSVA).

MSVA is defined as the distance from C7 to PNP-LINE, which can reflect the real sagittal imbalance. (Fig.2). PNP-LINE is the parallel line of TPT-LINE drawn from the posterior superior corner of the sacrum (Fig.2). In the process of compensation, PT increases. The PT value at this time is also a value after pelvic compensation. Vialle et al. used the formula PT $=0.37 \mathrm{PI}-7$ to get theoretical PT value (TPT) through linear regression analysis to evaluate compensation of pelvis [7]. The TPT value means the real PT value without pelvic compensation. The difference between the TPT value and PT value is defined as $\triangle P T$ ,which can reflect the pelvic rotation compensation. According to the TPT value, the line drawn from the 
midpoint of the hip axis is defined as TPT-LINE. Therefore, the author proposes a hypothesis: the MSVA can better reflect the sagittal balance.

In this study, the spine-pelvic parameters LL, TK, TLK, SS, PI, and quality of life scores were significantly improved postoperatively, indicating that orthopedic surgery can significantly improve spine-pelvic alignment and the patient's quality of life of patients with ASD. Meanwhile, we found that postoperative quality of life score was correlated with postoperative SVA and MSVA (Table 4). And MSVA had the stronger correlation with postoperative quality of life score. Therefore, we thought MSVA can be used as a new parameter to assess sagittal balance in patients with ASD. For patients with spinal deformity, satisfactory sagittal alignment is closely related to satisfactory function postoperatively. MSVA has important clinical significance for assessing the postoperative sagittal balance and the quality of life.

Traditional SVA can reflect the overall sagittal balance, but cannot describe the compensation of the pelvis. Pelvis compensation is not only related to a satisfactory spinal alignment, but also related to the patient's quality of life. MSVA is the parameter which can reflect the rotation of the pelvis and the overall sagittal balance of the spine simultaneously. Although, SVA is better than MSVA in ODI scores, MSVA has stronger correlation and higher significance than SVA in SRS22 scores. Especially for patients with severe pelvic compensation, SVA value may be satisfactory, but the quality of life is poor. In the case, the MSVA is huge which may imply the poor quality of life. In Fig 3, we can find that the SVA is satisfactory but MSVA is huge. In the case, the quality of life is not satisfactory. And orthopedic surgery can reduce MSVA and improve the quality of life of patients. However, we can't simply draw a conclusion that MSVA is better than SVA. MSVA can be regarded as a powerful supplement to assess the sagittal balance of spine and pelvis.

There are still many deficiencies in this study. There were no meaningful results regarding preoperative MSVA and SVA. The reason may be that the sample size of this retrospective study is limited, and the inclusion criteria did not exclude patients with lumbar spinal stenosis which can interfere with the research results. As an innovative spine-pelvic parameter, MSVA still need further study. In the next step, we hope to get the best range of MSVA by large samples to guide the clinical treatment.

\section{Abbreviations}

\begin{tabular}{|ll|}
\hline ASD & adult spinal deformity \\
\hline SVA & sagittal vertical axis \\
\hline MSVA & Modified sagittal vertical axis \\
\hline TPT & theoretical PT value \\
\hline PNP & Pelvis neutral position \\
\hline
\end{tabular}

\section{Declarations}


Acknowledgements

The authors would like to thank all staff from the participating departments and clinics.

\section{Funding}

There was no financial support for the study.

\section{Author information}

Yang Yu and Bing Wu contributed equally to this work and are considered co-first authors.

\section{Affiliations}

Nankai university school of medicine, Tianjin, 100071, China

Dr. Yang Yu

Chinese People's Liberation Army General Hospital, Fuxing Road, Haidian District, Beijing, 100853, China

Dr. Yang Yu, Dr. Bing Wu, Dr. Zhaohan Wang, Dr. Junyao Cheng, Dr. Bo Li, Miss. Jinjin Liu, Dr. Kai Song \&Dr. Zheng Wang

\section{Contributions}

Dr. Yang Yu and Dr. Bing Wu analyzed data and drafted the main manuscript text. Dr. Kai Song was responsible to correct and revise the manuscript. Dr. Zhaohan Wang, Dr. Junyao Cheng, Dr. Bo Li, Miss. Jinjin Liu were responsible to collect data. Dr. Zheng Wang were responsible to the idea and direction. All authors read and approved the final manuscript. Acknowledgements

\section{Corresponding authors}

Correspondence to Kai Song and Zheng Wang.

\section{Ethics declarations}

This retrospective review study involving human participants was in accordance with the ethical standards of the institutional and national research committee and with the 1964 Helsinki Declaration and its later amendments or comparable ethical standards. The study was approved by the medical ethics committee of our hospital. Informed consent was obtained from all individual participants included in the study.

\section{Consent for publication}

Not applicable 


\section{Competing interests}

There is no competing interest.

\section{Availability of data and materials}

Not applicable.

\section{References}

1. Kotwal S, Pumberger M, Hughes A, et al. Degenerative scoliosis: a review[J]. HSS J, 2011, 7(3):257264.

2. Garcíaramos CL, Obilchavarría CA, Záratekalfópulos B, et al. Degenerative adult scoliosis[J].Acta Ortop Mex, 2015, 29(2):127-138.

3. Aebi M. The adult scoliosis[J]. Eur Spine J, 2005, 14(10):925-948.

4. Barrey C, Roussouly P, Perrin G, et al. Sagittal balance disorders in severe degenerative spine. Can we identify the compensatory mechanisms[J]? Eur Spine J, 2011, 20(Suppl 5):S626-633.

5. Baghdadi YM, Larson AN, Dekutoski MB, et al. Sagittal balance and spinopelvic parameters after lateral lumbar interbody fusion for degenerative scoliosis: a case-control study[J]. Spine, 2014, 39(3):166-73.

6. Schwab F, Lafage V, Patel A, et al. Sagittal plane considerations and the pelvis in the adult patient[J]. Spine, 2009, 34(17): 1828-1833

7. Vialle, Raphal. Radiographic analysis of the sagittal alignment and balance of the spine in asymptomatic subjects.[J]. Journal of Bone \& Joint Surgery American Volume, 2005, 87(2):260.

8. Tsai TH, Huang TY, Lieu AS, et al. Functional outcomeanalysis: instrumented posterior lumbar interbody fusion fordegenerative lumbar scoliosis[J]. Acta Neurochir, 2011, 153(3):547-555.

9. Silva FE, Lenke LG. Adult degenerative scoliosis: evaluationand management[J]. Neurosurg Focus, 2010, 28(3): E1.

\section{Tables}

Table 1 comparison of spine-pelvic parameter preoperatively and postoperatively 


\begin{tabular}{llll} 
& preoperative & postoperative & $\mathrm{P}$ \\
\hline $\mathrm{TK}\left(^{\circ}\right)$ & $14.42 \pm 13.57$ & $23.76 \pm 12.75$ & 0.00 \\
\hline $\mathrm{TLK}\left(^{\circ}\right)$ & $19.23 \pm 18.11$ & $12.17 \pm 11.8$ & 0.02 \\
\hline $\mathrm{LL}\left(^{\circ}\right)$ & $22.43 \pm 18.52$ & $37.52 \pm 12.16$ & 0.00 \\
\hline $\mathrm{SS}\left(^{\circ}\right)$ & $18.25 \pm 15.23$ & $26.60 \pm 9.297$ & 0.00 \\
\hline $\mathrm{PT}\left({ }^{\circ}\right)$ & $28.70 \pm 1.45$ & $20.39 \pm 1.18$ & 0.00 \\
\hline $\mathrm{PI}\left({ }^{\circ}\right)$ & $47.47 \pm 14.3$ & $47.40 \pm 13$ & 0.97 \\
\hline $\mathrm{PI}-\mathrm{LL}\left({ }^{\circ}\right)$ & $25.05 \pm 21.9$ & $9.87 \pm 13.3$ & 0.00 \\
\hline $\mathrm{PI} / \mathrm{LL}$ & $5.90 \pm 26.06$ & $1.41 \pm 0.62$ & 0.19 \\
$\mathrm{SVA}(\mathrm{mm})$ & $59.09 \pm 61.2$ & $34.35 \pm 28.4$ & 0.01 \\
\hline $\mathrm{RSVA}(\mathrm{mm})$ & $205.9 \pm 94.7$ & $131.4 \pm 58.6$ & 0.00
\end{tabular}

Table 2 Comparison of quality of life scores in patients with ASD preoperatively and postoperatively

\begin{tabular}{llll} 
& preoperative & postoperative & $\mathrm{P}$ \\
\hline ODI & $51.06 \pm 10.51$ & $24.29 \pm 16.69$ & 0.00 \\
\hline Lumbar pain VAS & $7.28 \pm 3.49$ & $1.71 \pm 2.40$ & 0.00 \\
\hline Leg pain VAS & $3.56 \pm 4.22$ & $1.08 \pm 2.34$ & 0.00 \\
\hline SRS22 & $33.67 \pm 5.75$ & $53.50 \pm 9.45$ & 0.00
\end{tabular}

Table 3 Correlation analyses between preoperative quality of life scores and spine-pelvic parameters

\begin{tabular}{|lllllll|}
\hline & PI & PT & LL & PI-LL & SVA & MSVA \\
\hline ODI & 0.229 & 0.020 & 0.005 & 0.089 & 0.049 & 0.113 \\
\hline SRS22 & -0.219 & -0.130 & -0.162 & 0.003 & -0.216 & -0.140 \\
\hline
\end{tabular}

Table 4 Correlation analyses between postoperative quality of life score and spine-pelvic parameters

\begin{tabular}{|lllllll|}
\hline & PI & PT & LL & PI-LL & SVA & MSVA \\
\hline ODI & 0.205 & 0.154 & -0.047 & 0.243 & $0.329^{*}$ & $0.266^{*}$ \\
\hline SRS22 & -0.137 & -0.13 & 0.208 & $-0.324^{*}$ & $-0.284^{\star}$ & $-0.338^{\star *}$ \\
\hline
\end{tabular}


${ }^{*} \mathrm{p}<0.05,{ }^{* *} \mathrm{p}<0.01$

\section{Figures}
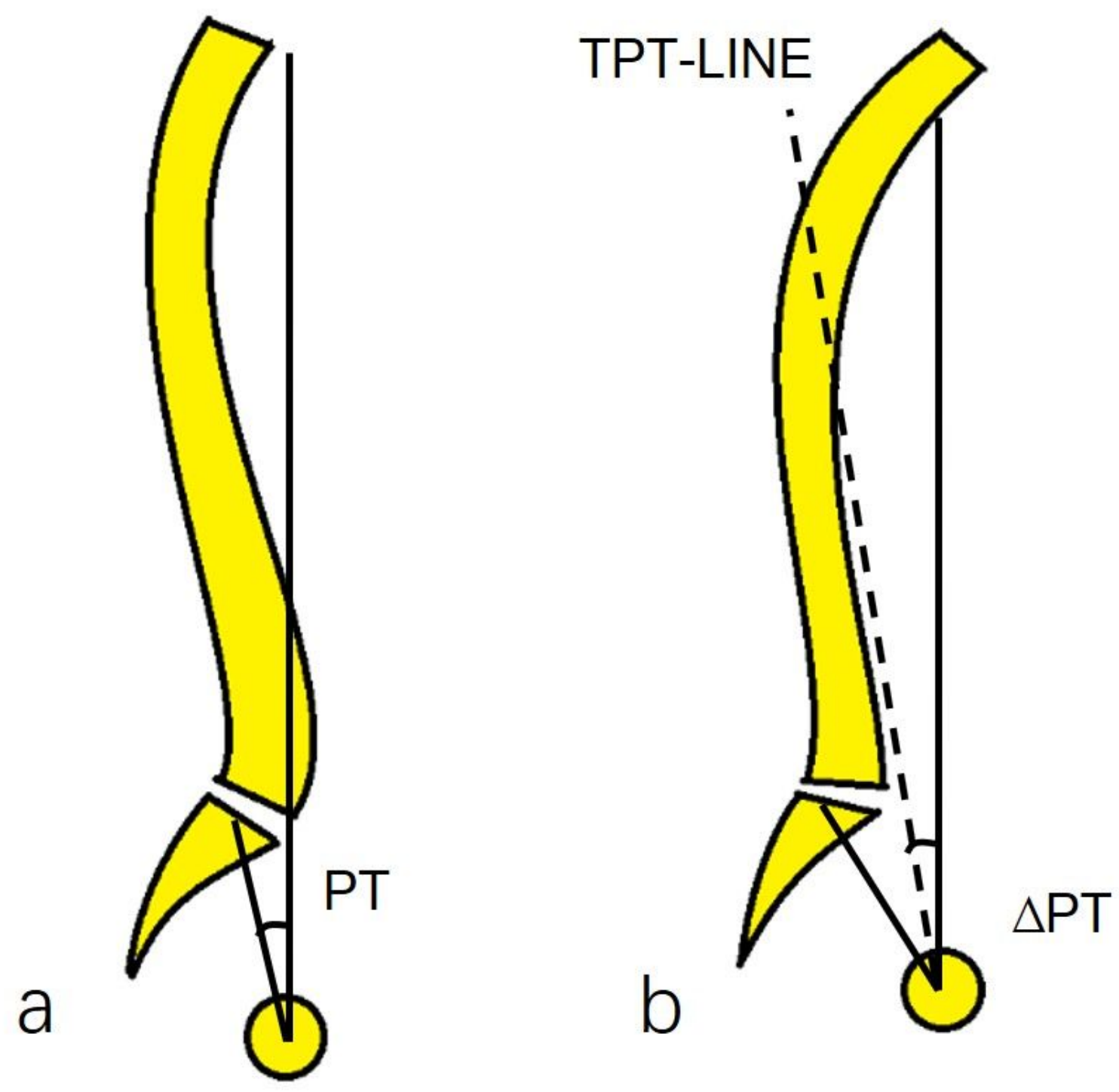

Figure 1

a. When there is no pelvic compensation, the PT value is the real PT value; $b$. With the increase of age, the lumbar lordosis decreased, the body shifted forward trend was obvious. In the case, the pelvic rotated backward to compensate, and the PT value increased. The PT value at this time is a value after pelvic compensation. Through the formula: theoretical PT $=0.37 * \mathrm{PI}-7$, the theoretical PT (TPT) value of patients with pelvic compensation can be calculated. The difference between the TPT value and PT value is defined as $\triangle P T$, which can reflect the pelvic rotation compensation. According to the TPT value, the line drawn from the midpoint of the hip axis is called is called TPT-LINE (The dotted line). 


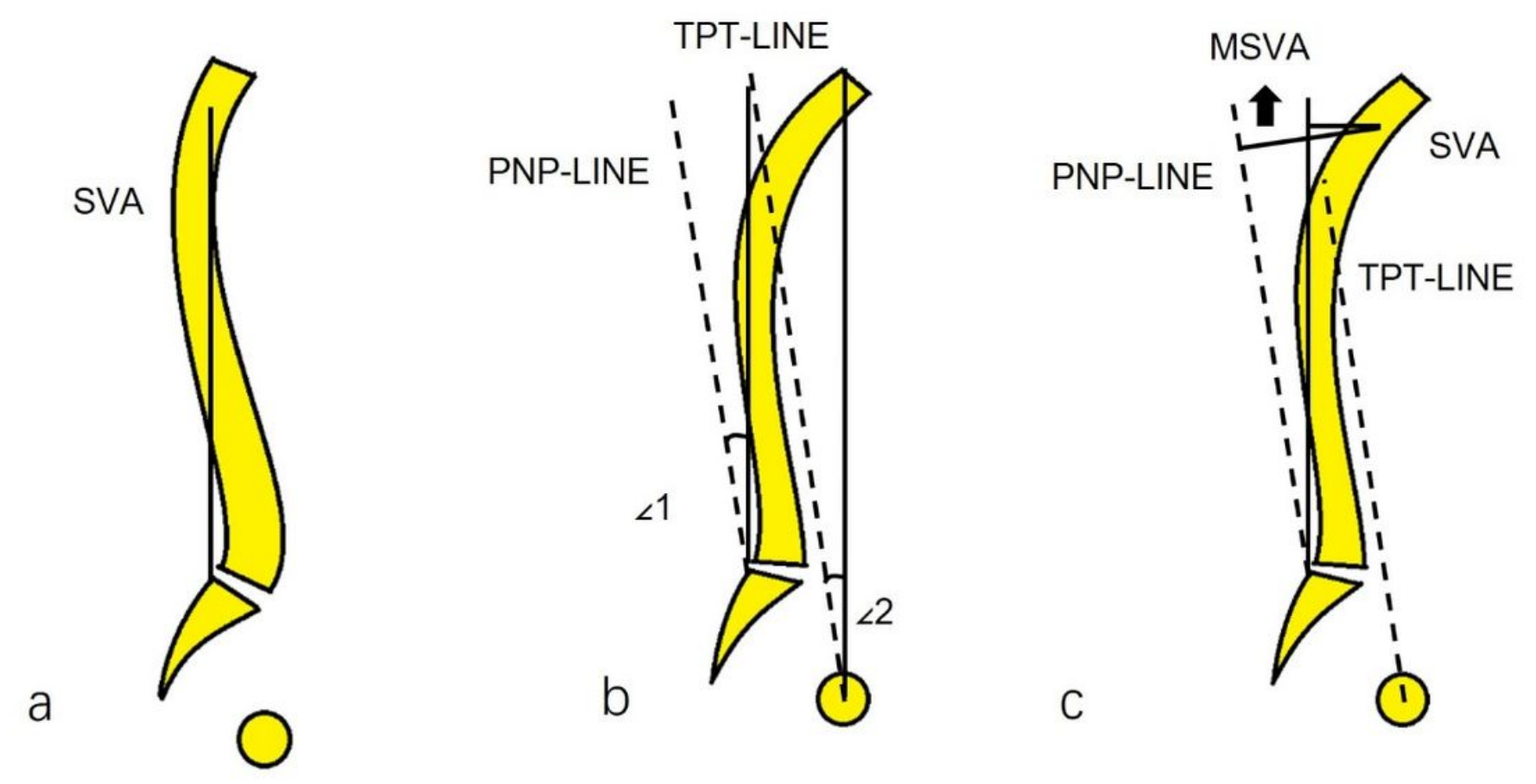

Figure 2

a. The C7 plumb line should fall on the posterior upper corner of the sacrum, that is SVA = 0; b. PNP-LINE is the parallel line of TPT-LINE drawn from the posterior superior corner of the sacrum, $\angle 1=\angle 2=\Delta \mathrm{PT}$; c. The distance between $\mathrm{C} 7$ point and PNP-LINE can reflect both sagittal balance and pelvic compensation, which is called modified SVA. 


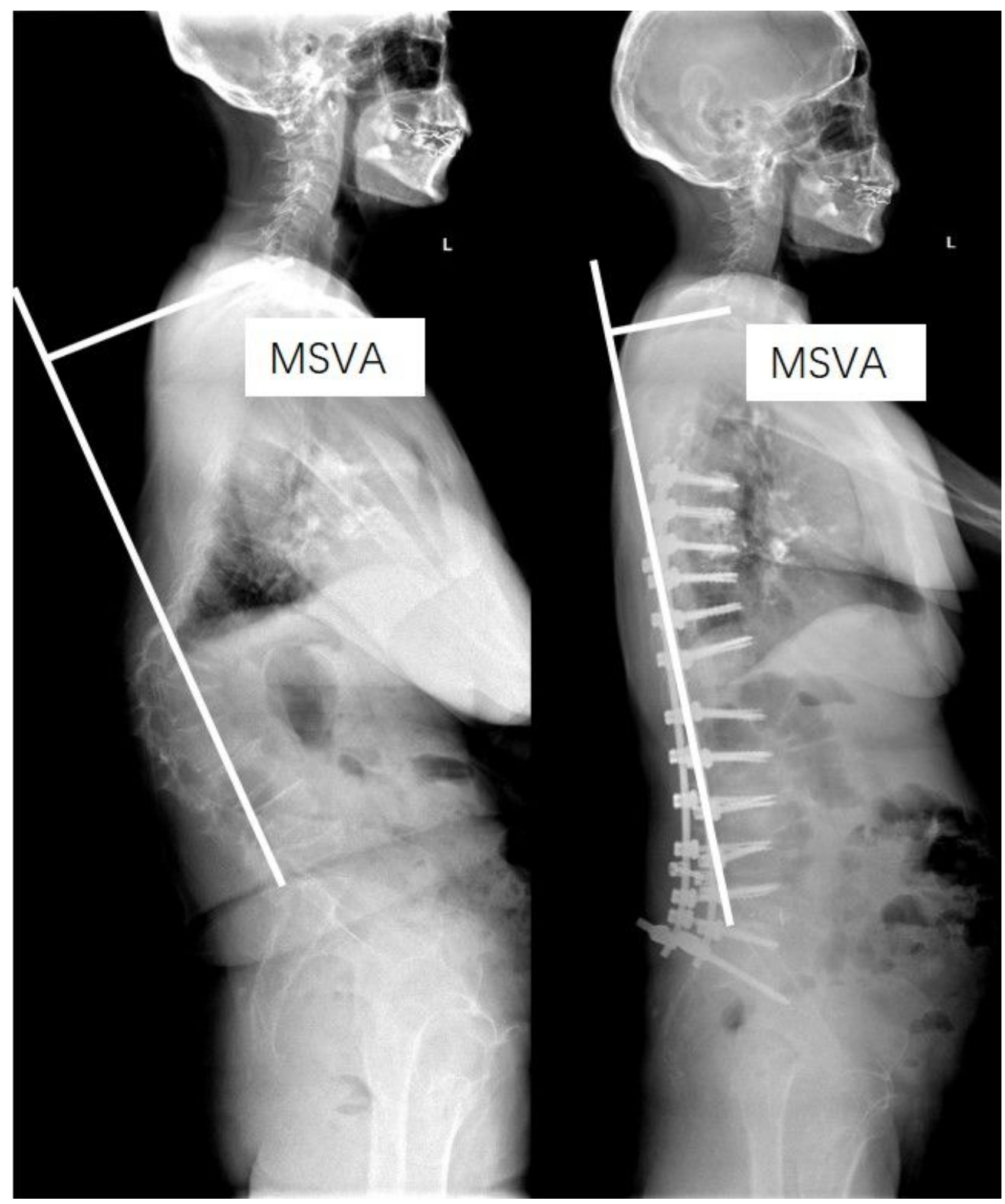

Figure 3

A 65 years old female patient, pre-operation SVA is $0 \mathrm{~mm}$, pre-operation MSVA is $234 \mathrm{~mm}$, pre-operation ODI score is 54, pre-operation SRS22 score is 32, pre-operation low back VAS score is 10; post-operation SVA is $0 \mathrm{~mm}$ post-operation MSVA is $134 \mathrm{~mm}$, post-operation ODI score is 36 , post-operation SRS22 score is 47 , post-operation low back VAS score is 0 . 